\title{
Density functional study of manganese atom adsorption on hydrogen-terminated armchair boron nitride nanoribbons
}

\begin{abstract}
In this paper, we have investigated stable structural, electric and magnetic properties of manganese $(\mathrm{Mn})$ atom adsorption on armchair hydrogen edge-terminated boron nitride nanoribbon (A-BNNRs) using first principles method based on density-functional theory with the generalized gradient approximation. Calculation shows that $\mathrm{Mn}$ atom situated on the ribbons of A-BNNRs is the most stable configuration, where the bonding is more pronounced. The projected density of states (PDOS) of the favored configuration has also been computed. It has been found that the covalent bonding of boron (B), nitrogen $(\mathrm{N})$ and $\mathrm{Mn}$ is mainly contributed by $\mathrm{s}, \mathrm{d}$ like-orbitals of $\mathrm{Mn}$ and partially occupied by the $2 \mathrm{p}$ likeorbital of $\mathrm{N}$. The difference in energy between the inner and the edge adsorption sites of ABNNRs shows that Mn atoms prefer to concentrate at the edge sites. The electronic structures of the various configurations are wide, narrow-gap semiconducting and half-metallic, and the magnetic moment of $\mathrm{Mn}$ atoms are well preserved in all considered configurations. This has shown that the boron nitride $(\mathrm{BN})$ sheet covered with $\mathrm{Mn}$ atoms demonstrates additional information on its usefulness in future spintronics, molecular magnet and nanoelectronics devices.
\end{abstract}

Keyword: Boron nitride nanoribbons; Density functional theory; Structural; Electric and magnetic properties 